\title{
Philosophiques
}

\section{L’idéalisme est-il spiritualiste?}

\section{Roch Bouchard}

\section{Volume 12, numéro 1, printemps 1985}

URI : https://id.erudit.org/iderudit/203271ar

DOI : https://doi.org/10.7202/203271ar

Aller au sommaire du numéro

\section{Éditeur(s)}

Société de philosophie du Québec

ISSN

0316-2923 (imprimé)

1492-1391 (numérique)

Découvrir la revue

Citer cet article

Bouchard, R. (1985). L’idéalisme est-il spiritualiste? Philosophiques, 12(1), 53-71. https://doi.org/10.7202/203271ar

\section{Résumé de l'article}

Cet article est une réflexion sur le rapport entre deux courants de la philosophie classique, l'idéalisme postkantien et le spiritualisme venu de Descartes, tels qu'ils se rencontrèrent dans l'oeuvre de J. Lachelier. Il étudie pourquoi on attendit du postkantisme qu'il apportât une évidence affermie aux propositions du spiritualisme, lors même que la critique kantienne eut mis en crise la méthode qui les justifiait rationnellement, et bien qu'on dût laisser hors du système nouveau des idées aussi importantes que celles de la survie et du Dieu transcendant, lesquelles étaient renvoyées à la croyance religieuse. L'analyse menée ici du concept d'idéalisme invite cependant à juger que, même affirmées comme croyances, ces idées ne pouvaient être que résolutoires pour un système qui commence par la négation d'un au-delà de la pensée. 
PHILOSOPHIQUes, Vol. XII, Numéro 1, Printemps 1985

\title{
L'IDÉALISME EST-IL SPIRITUALISTE ?*
}

\author{
par Roch Bouchard
}

RÉSUMÉ. Cet article est une réflexion sur le rapport entre deux courants de la philosophie classique, l'idéalisme postkantien et le spiritualisme venu de Descartes, tels qu'ils se rencontrèrent dans l'œuvre de J. Lachelier. Il étudie pourquoi on attendit du postkantisme qu'il apportât une évidence affermie aux propositions du spiritualisme, lors même que la critique kantienne eut mis en crise la méthode qui les justifiait rationnellement, et bien qu'on dût laisser hors du système nouveau des idées aussi importantes que celles de la survie et du Dieu transcendant, lesquelles étaient renvoyées à la croyance religieuse. L'analyse menée ici du concept d'idéalisme invite cependant à juger que, même affirmées comme croyances, ces idées ne pouvaient être que résolutoires pour un système qui commence par la négation d'un au-delà de la pensée.

ABSTRACT. This article is a reflection on the relation between two currents of classical philosophy, postkantian idealism and cartesian spiritualism, as they are found in the work of J. Lachelier. It studies why it was expected that postkantian idealism would bring strong evidence in support of the spiritualist affirmations, even though the kantian critique placed the method that traditionaly justified those affirmations in a state of crisis, and even though ideas as important as those of an afterlife and of a transcendant God where excluded from this new system and taken over by religious belief. However, the analysis of the concept of idealism invites us to think that, even as beliefs, such ideas will always remain redhibitory for a system that begins with the negation of anything beyond thought.

Au siècle dernier se constituèrent les philosophies qui pour l'histoire représentent les formes classiques de l'idéalisme et du spiritualisme. L'idéalisme classique, ou absolu, est né en Alle-

* Une première rédaction de cet article a déjà paru dans la revue Carrefour, Ottawa, décembre 1982, pp. 3-23. 
magne des prolongements que donnèrent au kantisme les systèmes de Fichte, Schelling, Hegel. Son point de vue méthodologique est celui d'une raison qui considère le monde comme son déploiement. Le "spiritualisme " est une tradition de philosophie française qui va, peut-on dire, de Maine de Biran à Jean Nabert. Son point de vue méthodologique, qui est celui de la connaissance de soi, n'aura pas libéré l'ampleur de spéculation, la hardiesse quasi démiurgique de l'idéalisme allemand. Du moins le spiritualiste se fit-il fort de mesurer ses évidences, de recueillir avec grande attention les faits intérieurs et ce qu'ils pouvaient donner à penser du reste.

Cette assez bonne conscience s'est trouvée en crise lorsqu'on a pris en compte ce que Kant avait dit de la portée véritable de toute expérience. Que l'analyse réflexive nous renseignât sur des réalités, voilà qui se mettait à faire question. Le demi-idéalisme de Kant se présentait donc au spiritualisme comme une réfutation. Mais il amenait avec lui, et peut-être aussi en lui, l'idéalisme absolu des postkantiens, en lequel le spiritualiste perplexe crut trouver le moyen d'une reformulation. Jules Lachelier, qui vécut exemplairement la crise, fut celui qui introduisit l'idéalisme dans l'université française. "J'ai combattu comme vous . . . pour le spiritualisme, écrira-t-il à un disciple de Victor Cousin, car vous m'accorderez bien que l'idéalisme est une des formes du spiritualisme ${ }^{1}$.

Comment l'idéalisme entraîna ce renouvellement du spiritualisme, je voudrais cependant qu'on le regarde de plus près, et qu'on examine dans quelle mesure le spiritualisme y gagna vraiment de devenir plus rationnel. Car je voudrais montrer qu'à raisonner strictement, le cadre idéaliste ne convenait au spiritualisme que bien relativement, et même qu'il lui répugnait pour une part essentielle de ses thèses, contrairement à une opinion répandue qui va jusqu'à désigner une philosophie par l'autre. Autant que l'on puisse ramener à deux articles également importants les thèses du spiritualisme classique, l'esprit existe, l'au-delà existe, on devrait voir ici qu'il n'était que pour moitié réceptif à l'idéalisme. Je veux prouver en tout cas que les deux articles n'y pouvaient trouver leur compte. Le premier y reçut

1. À Paul Janet, 15 novembre 1885, Lettres, Paris, hors-commerce, 1933, p. 133. 
certes une conceptualisation puissante, mais le second, même renvoyé à la croyance religieuse, ne pouvait représenter pour le nouveau système qu'une contradiction rédhibitoire.

Ces conclusions sont censées ressortir de l'étude de la rencontre de l'idéalisme et de son initiateur français, laquelle occupera la plus grande partie de ce travail. J'y considérerai les principales mises en question dont le spiritualisme fit l'objet avec l'avènement du kantisme, par quelles transformations il a pu assimiler cette philosophie, et comment Lachelier crut conserver quelque existence doctrinale aux idées de Dieu et de la survie, bien que le système transformé fût jugé incapable de les accueillir. Jusqu'à quel point l'idéalisme est théoriquement un spiritualisme, il me semble qu'on l'apercevra alors assez clairement.

***

Pour Lachelier, le kantisme met le spiritualisme dans de graves difficultés, mais aussi, dans la mesure où il contient le germe du postkantisme, il marquera sa régénération. Qu'est-ce que Kant se trouve à reprocher au spiritualisme ? Essentiellement sa méthode, comme on a dit, et sa prétention à connaître l'existence de Dieu.

D'abord sa méthode. Dans la tradition française depuis Descartes, et singulièrement depuis Maine de Biran, la certitude souveraine porte sur ce qui est éprouvé au tout dedans de nous. L'indubitable absolu, on le trouve à ce niveau d'intimité, ou de subjectivité, dont nous ne pouvons même dissocier le sentiment de nous-mêmes ; et toute autre existence par delà cette sphère inétendue de notre moi ne sera affirmable qu'en s'articulant à l'évidence originelle. C'est une idée omniprésente chez les mầtres français du spiritualisme. Dans l'ontologie de Maine de Biran, les catégories dans lesquelles nous concevons les choses, la certitude même du monde extérieur, tirent leur origine de l'expérience de l'effort, qui est le fond de la conscience humaine. Félix Ravaisson, qui fut le maître de Lachelier, pense à peu près de même, mais sous l'effort il voit le désir comme substance de la psyché, et sous le désir il pose l'amour comme seul principe de surélévation du désir dans l'effort, se mettant par là en position d'induire une métaphysique panpsychiste, où toute chose 
est émanation d'une divinité généreuse d'elle-même. Victor Cousin n'intellige pas le monde dans sa conscience, mais du moins y voit-il le phénomène crédible de l'esprit, et y entend même, proférations de la Raison universelle, ces prémisses de la philosophie spiritualiste que sont pour lui les principes de substance et de causalité. Autant de doctrines, donc, qui se développent, malgré des différences parfois marquées, par l'analyse des contenus de la conscience. Or ce que Kant apprend ici à Lachelier, c'est que l'autorité du sens intime ne suffit en aucun cas à une conclusion solide. Puisqu'en effet nous ne connaissons que ce que nous sentons, et ne sentons que des phénomènes, c'est-à-dire des sous-produits de notre contact avec la réalité ; puisque les conditions mêmes de notre connaissance nous empêchent à jamais de connaître le fond des choses, il devient évidemment illusoire de saisir le moi, ou l'esprit, ou la liberté, dans ce qui n'en est qu'apparence. "C'est en vain, écrit Lachelier, qu'on en appellerait, contre le matérialisme, au sentiment immédiat de l'action spirituelle, car ce que nous sentons n'est pas en question, mais il s'agit de savoir si ce que nous sentons en nous-mêmes est, comme ce que nous voyons, fondé en raison, intrinsèquement vrai et intelligible, conforme à une idée : il faut déduire l'action spirituelle ${ }^{2}$. Il faut revoir la façon dont l'esprit est connu.

L'autre grief venu de Kant concerne l'aptitude de la philosophie à établir spéculativement l'existence de Dieu. Pour Biran et pour Cousin, Dieu s'atteignait comme cause. Pour Ravaisson, il était implicitement présent à la conscience ${ }^{3}$. Contre les premiers, Kant met au jour le paralogisme qui consiste à remonter du monde à Dieu par le principe de causalité. "Sans doute, enseignera de même Lachelier, dont on sait que, professeur à l'École normale, il gardait ouverte sur sa table la Critique de la raison pure, sans doute tout effet suppose une cause, mais une cause de même ordre que lui ; c'est du moins tout ce que nous sommes en droit d'affirmer d'après les lois de notre entendement». Que je remonte au plus lointain des causes qui s'enchaînent en ce monde, "j'irai toujours de l'homogène à l'ho-

2. Au même, 12 décembre 1887, Id., p. 140.

3. Dieu est " en nous ", " caché par cela seul qu'il est trop au dedans, et dans ce fonds intime de nous-mêmes où nous ne descendons pas ". (De l'Habitude, P.U.F., 1957, p. 53). 
mogène, et jamais je n'atteindrai le bout de cette chaîne infinie $"{ }^{4}$. Comprenons que le principe de causalité, dès lors qu'il vaut seulement pour l'ordre phénoménal, dont il est une forme a priori, se retrouve, devant l'inférence à un au-delà de l'expérience, comme un pont suspendu à une seule rive. Ce principe, a dit Kant, « n'a de valeur que dans le champ de l'expérience et . . hors de ce champ, il serait entièrement détourné de sa destination. (. . .) Pour que la loi de la causalité, valable dans l'expérience, pût convenir à l'être premier, il faudrait que cet être fût partie de la chaîne de l'expérience ; mais alors il serait lui-même, à son tour, conditionné, comme tous les phénomènes $»^{5}$. Prouver Dieu par la causalité, c'est le donner pour relatif, c'est nier qu'il soit Dieu.

Quant à l'idée ravaissonnienne que Dieu se manifeste à quelque fond de la conscience, Kant apprend à Lachelier que l'absolu dont il nous est permis de prendre conscience ne peut être pour le philosophe qu'une idée, qu'une simple forme dont nulle théodicée ne saurait s'autoriser : «Ce que j'ai cru remarquer en lisant Kant, c'est que nous ne pouvons avoir conscience que d'un infini formel ou de pensée, et que l'infini matériel, ou d'existence, est aussi nécessairement étranger à notre conscience qu'il est distinct de l'univers " ${ }^{6}$.

Il apparaîtra dans ces conditions que la meilleure justice que la philosophie peut rendre à la croyance religieuse est de l'affranchir de tous ces discours, de toutes ces supposées évidences qui finalement l'encombrent et la démentent. Il faudra que sur le Dieu transcendant du christianisme le silence soit tel qu'on puisse y croire même si la philosophie ne le rejoint pas, même si elle s'arrête plutôt au panthéisme, comme fera celle de Lachelier, nous le verrons, qui présentait son cours de théodicée en déclarant : "Notre théodicée n'aura pour but ni d'établir, ni de réfuter le panthéisme, mais de prouver qu'il exprime fidèlement le contenu de la conscience, et de faire sentir par là le besoin d'une croyance qui le dépasse $" 7$.

4. Cours de logique, leçon XV. Les Cours de Lachelier, recueillis par des étudiants, furent donnés de 1864 à 1885 à l'École normale supérieure de Paris, et sont conservés manuscrits à la Bibliothèque de l'Université de Paris.

5. Critique de la raison pure, trad. A. Tremesaygues et B. Pacaud, P.U.F., 1965, p. 449.

6. À Ravaisson, 15 août 1868 , Lettres, p. 58.

7. Cours de théodicée, introduction. 
Le kantisme a donc mis en lumière l'inanité des arguments par lesquels la métaphysique française justifiait l'affirmation de l'esprit et de l'au-delà. Mais comment il a pu renouveler le spiritualisme, et même le relancer vers des sommets nouveaux, il faut le voir depuis cet endroit de la Critique de la raison pure où il est traité des « principes de la possibilité de l'expérience ".

Kant y explique (et c'est à cette page, nous le savons par Émile Boutroux ${ }^{8}$, que le livre était ouvert sur la table de Lachelier) que nos représentations ne sont des représentations pour nous, ne nous sont réelles comme moments d'une même séquence, comme fractions d'une même expérience, que si elles sont rapportées à un sujet identique à lui-même qui les pense. Dans tout fait de conscience se trouve enveloppée l'affirmation par lui-même d'un je pense, moyennant quoi est saisissable dans une même appréhension, est appropriable, ce pur senti qui autrement resterait une diversité toute fugitive et indicible. Ce je pense n'est pas le sujet psychologique, car ce dernier, loin de s'imposer aux sensations, en quelque sorte voyage sur elles, comme l'a observé Hume. Le je pense est simplement la fonction a priori spontanée par laquelle les phénomènes conviennent à notre connaissance. En cela consiste le moi transcendantal, foyer des catégories sous lesquelles s'organise le divers de l'expérience pour constituer la nature que nous connaissons ${ }^{9}$. Ce qu'il y a par conséquent de connaissable dans les choses, c'est rigoureusement notre façon de les connaître. La forme du pensable est la forme du penser. Ainsi apprend-on de Kant à fonder le monde, comme dira Brunschvicg, "sur la structure d'un organisme intellectuel ${ }^{10}$. Une telle perspective est prometteuse : on ravalera difficilement l'esprit à la nature si, au lieu qu'il en soit un incompréhensible à-côté, enclave dans le mécanisme, tache noire de la rationalité, c'est bien plutôt la nature qui s'entend par lui, le dispensateur de toute intelligibilité accessible à nous.

À partir de là, l'autonomie de l'esprit n'est plus à chercher dans des introspections condamnées à l'apparence, ou dans des raisonnements à la Ravaisson qui réellement sont des suppu-

8. Nouvelles études d'bistoire de la philosophie, Alcan, 1927, p. 11.

9. Voir Critique de la raison pure, p. 107 et suiv.

10. Notice, GUVRES, 2 tomes, Alcan, 1933, I,' p. XIII. 
tations, mais dans la considération de ce fait que l'expérience de l'univers n'est possible que sous la détermination d'une pensée. On pourra même dire avec Ravaisson que la nature n'est que l'acte de l'Esprit, si, à la façon des postkantiens, on conçoit l'être corporel comme le dernier moment d'un processus dialectique qui consiste dans l'autoposition d'un moi, c'est-à-dire sa figuration dans un espace où il se reconnaît et dont à la fois il se distingue. C'est ce que dira Lachelier : « Le véritable Kant, pour moi, et le véritable représentant de sa philosophie de l'esprit, c'est Fichte ». "Il n'y a pas d'autre être que la pensée ou la vérité elle-même, qui, d'une part, se concentre et se réfléchit dans les esprits, et qui de l'autre enveloppe et enchaîne les corps, qui ne sont que les phénomènes des esprits " ${ }^{11}$. Il n'y a pas de matière, il y a l'acte de la phénoménalisation. "Ravaisson, a dit Gabriel Séailles, a donné à Lachelier le principe et la conclusion de sa philosophie : l'esprit est ce qu'il est. Kant lui a donné le sens de cette formule, la méthode qu'elle contient ${ }^{12}$.

Si toutefois Lachelier a lu cette formule chez Kant, ce fut dans l'esprit de la doctrine, et non dans le texte. Kant n'allait pas si loin dans la métaphysique, lui qui croyait avoir destitué toute connaissance d'un au-delà de l'expérience, y compris assurément celle qui verrait dans le sensible la diffraction de l'esprit. Le sujet détermine l'objet, mais ne le constitue pas, pensait-il, dans sa réalité. La réalité se passe d'autant mieux de nous qu'elle nous échappe entièrement. Aux yeux de Kant, donc, si le sensible n'a d'essence que par un moi non sensible, il reste que cet empire du sujet sur l'objet, de l'intelligible sur le sensible, qui ouvrirait peut-être un espace à la métaphysique, se réduit en fait, devant le réel intouchable, à quelque chose de purement formel, d'aussi mince qu'une image sur une lentille déformante.

La différence fondamentale qu'il faut apercevoir de Kant à Lachelier, et à l'ensemble du postkantisme, c'est le passage du second par l'idéalisme absolu, à savoir par la négation de l'ensoi. Chez Kant, la vraie convenance de l'idée d'être est à l'audelà du connu. Dès lors, quoi que l'analyse de la connaissance puisse déduire comme condition a priori de celle-ci, depuis la

11. À Denys Cochin, 10 octobre 1913, Lettres, p. 177-178.

12. La philosophie de Jules Lachelier, Vrin, 1935, p. 3. 
forme qui structure le dehors jusqu'au pur principe qui le pense sans se penser soi-même, c'est-à-dire ce moi transcendantal qui n'apparaît pas sur l'écran de l'expérience et n'appartient pas plus au monde de l'en-soi, rien donc de ce qui sera déduit comme cause que l'empirie est connaissable ne détiendra de réalité assignable. À parler absolument, le noumène est l'être, et le phénomène $y$ a part en tant qu'inversion du noumène. Mais le principe de la vérité de cette inversion, le transcendantal, quel est son statut ontologique ? S'il relève du phénomène, il n'est pas principe de vérité, car il est alors matière plutôt que forme. S'il relève du noumène, s'il s'enracine dans le moi substantiel sous le moi psychologique, il ne fonde pas non plus la vérité, car il y aurait selon cette hypothèse autant de vérités que de sujets connaissants, attendu que pour cette doctrine l'épreuve d'un modèle extramental n'est jamais permise. Vraiment, pour qu'il y ait la connaissance objective, il faut que le transcendantal soit unique comme la vérité, et donc qu'il soit indépendant de l'individualité nouménale autant que de la phénoménale. Mais quel serait donc l'être de cette chose universelle ? Nous avons ici la raison pour laquelle le kantisme selon Kant ne pouvait se développer en spiritualisme : en regard de l'en-soi, le transcendantal est sans statut ontológique.

Mais l'idéalisme absolu nie l'en-soi. Par cette opération décisive, il libère l'idée d'une pensée qui crée son objet, puisqu'en effet les phénomènes sont tout de même les phénomènes de quelque chose, et il dissipe l'ambiguïté sur l'unicité de cette pensée qui est la vérité, puisqu'il n'y a plus cette multiplicité nouménale, hors juridiction, de structures cognitives pour menacer l'unité de la vérité. Et ainsi impose-t-il une ontologie moniste du transcendantal, lequel devient la pensée universelle. Louis Millet a montré la nature profondément plotinienne de cette ontologie où le sensible est l'expression dégradée d'une pensée qui a l'unicité de l'absolu ${ }^{13}$.

Sans doute, cette pensée ou conscience universelle est théologiquement inintéressante. Car bien qu'elle constitue le monde, elle n'est rien en dehors de lui. Comme le moi transcendantal chez Kant, elle échappe à l'expérience, sinon elle n'en est pas

13. Le symbolisme dans la philosopbie de Lachelier, P.U.F., 1959. 
la règle. Donc elle est inconsciente à elle-même. Rien de son être ne précède son acte, qui est de s'apparaître dans. les phénomènes. Elle n'est pas une substance qui passe à l'acte, mais un acte absolu, une spontanéité, un pur principe. "Ne craignons pas, dit Lachelier, de suspendre en quelque sorte la pensée dans le vide ; car elle ne peut reposer que sur elle-même : le dernier point d'appui de toute vérité et de toute existence, c'est la spontanéité absolue de l'esprit ${ }^{14}$. Que l'esprit fournisse la matière et la forme du connaître qui est l'être, cela ne le pose donc pas comme une sorte de personnalité du monde. Comme explique notre auteur : "Il ne faut pas chercher l'esprit en dehors des faits qu'il informe et dans lesquels il se réalise. Il n'y a pas plus de substance pensante que de substance étendue. Je ne crois même pas qu'il faille parler de sujet absolu ni de pensée universelle, comme si la pensée préexistait aux choses ou pouvait être quelque chose en dehors d'elles. La pensée, c'est la vérité, et la vérité est dans les choses elles-mêmes. C'est là que l'esprit doit se chercher, s'il veut se trouver... " ${ }^{15}$.

L'esprit n'est rien pour lui-même, et pourtant il est bien réel, parce que, justement, le connaître est l'être. Si le point de vue substantialiste était possible, ou, si l'on veut, pour un hypothétique observateur au delà de cette totalité qu'est la pensée du monde, il est vrai que notre « réalité » n'existerait pas. Elle serait comme un rêve absurdement cohérent qui à chaque instant pourrait cesser. Mais comme rien n'existe que l'acte absolu de la manifestation, il faut reconnaître à l'esprit et aux phénomènes, au manifesté et au manifestant, la totale extension du concept d'être. Il n'existe pas d'autre réalité pour disqualifier la nôtre, qui est comme une réalité par défaut, une idée-chose berkeleyenne. Et assurément, des deux termes de la manifestation, c'est l'esprit le plus réel, le strict analogué de l'être, autant que le sujet est entièrement principe de l'objet. "Si le monde existe parce qu'il est pensé, dit Lachelier, la pensée existe bien plus elle-même et, en quelque sorte, fait exister tout le reste. La pensée n'est pas un être, elle est l'être même " ${ }^{16}$.

14. Psychologie et métaphysique, GEUVRS, I, p. 208.

15. Société française de philosophie (S.F.P.), séance du 25 février 1904, CEUVRES, II, p. 126.

16. Cours de logique, leçon XV. 
La doctrine de la spiritualité de la conscience se voit ainsi reformulée dans des termes bien nouveaux pour la tradition française : la conscience n'est plus « en dehors et au-dessus du monde extérieur, mais au-dessous et au centre même de ce monde, qui n'en est . . . que l'épanouissement $"{ }^{17}$. Cela grâce à Kant, dont l'idée d'un a priori qui conditionne l'expérience a été absolutisée par le rejet de l'au-delà de l'a priori et de l'expérience. « Le seul tort de Kant, dit Lachelier, est de laisser subsister, au delà des phénomènes, la possibilité de la chose en soi (. . .). Il me semble au contraire que la nécessité causale, l'espace, le temps, c'est la nature elle-même . . ., que l'affirmation actuelle de l'être, c'est l'acte créateur lui-même, absolument efficace en même temps que purement idéal. Kant n'a fait que rouvrir à la philosophie un chemin depuis trop longtemps abandonné ${ }^{18}$.

Précisons que ce chemin conduisait à l'affirmation dé l'esprit, non à celle du Dieu transcendant. Cette seconde affirmation n'appartient pas à la philosophie, pense Lachelier à la suite de Kant, comme on a vu. Elle doit dépendre d'un acte de foi, qu'il juge d'ailleurs compatible avec le système, et même complémentaire de l'ensemble de la démarche philosophique. Une certaine doctrine la justifie donc. Elle dit que même si la philosophie est nécessairement panthéiste, notre intelligence et notre volonté ne sont pleinement intelligibles que si au panthéisme se superpose, sans communiquer avec lui, la croyance à l'au-delà religieux, attendu qu'il n'y a " pas de contradiction entre l'idée d'une nature complète en elle-même et celle d'un au-delà qui est à son égard comme s'il n'était pas " ${ }^{19}$.

Que la philosophie soit nécessairement panthéiste et que la croyance religieuse s'y surajoute sans la troubler, Lachelier le résume à l'article panthéisme du Vocabulaire de Lalande :

17. Psychologie et métaphysique, p. 199-200.

18. À Frédéric Rauh, 19 mars 1893, Lettres, p. 155-156. "Sans doute, dit Victor Delbos, en opposant aux prétentions théoriques de l'esprit la réalité certaine autant qu'inconnaissable de la chose en soi, le kantisme ferme la voie à une métaphysique qui prétendrait tout déduire d'un premier principe : mais qu'est-ce dans la doctrine de Kant que la chose en soi, sinon l'objet d'une affirmation injustifiable, en contradiction avec les exigences de la critique ? Et une fois la doctrine débarrassée de cet inutile résidu, ne trouvons-nous pas en elle de quoi la pousser tout naturellement vers une métaphysique nouvelle ? " $(\mathrm{De}$ Kant aux postkantiens, Aubier, 1940, p. 37-38).

19. S.F.P., 19 novembre 1908, EUVRES, p. 164. 
Le panthéisme . . . suppose d'abord l'unité de l'être, et ensuite que cet être est au fond spirituel, raison, liberté même, quoique d'abord sans conscience, mais destiné à s'apparaître à la fin à lui-même sous la forme de la pensée. Je ne crois pas qu'il y a d'autre être que celui-là pour la pure philosophie, et qu'elle est essentiellement panthéiste. Mais on peut croire à un au-delà du monde, spirituel aussi, mais sans mélange de matière, sans devenir, transréel, et inconnaissable pour nous dans nos conditions actuelles d'existence. Si l'on réserve pour cet au-delà le nom de Dieu, on se contentera d'appeler monde l'être spirituel universel ; et distinguant ainsi le monde de Dieu, on ne sera pas panthéiste ${ }^{20}$.

La philosophie est panthéiste, d'une part, parce que la pensée universelle que rien ne précède, donc qui se commence ellemême et son contenu, a l'attribut divin de l'aséité. Dans la logique intemporelle de cette causation de soi, la pensée est d'abord "l'idée de l'être ou de la vérité ", ou encore " la forme même de l'existence ", comme il est expliqué dans des pages difficiles et célèbres ${ }^{21}$. La philosophie est panthéiste, d'autre part, parce que cette pensée du monde, qui n'est pas effective sans son contenu, est avec lui dans un rapport de stricte immanence. « La pensée c'est la vérité, et la vérité est dans les choses elles-mêmes ». Mais on peut croire à un au-delà du monde, cela n'est pas incohérent, dit Lachelier. D'une manière plus positive, maintenant, pourquoi serait-ce rationnel ? Parce que, nous estil dit, cette existence a pour elle des indices objectifs, ainsi que je vais en résumer la thèse.

Réfléchissant sur le pari de Pascal, Lachelier conteste que de ce que l'on ne sache pas que Dieu existe, ni qu'il n'existe pas, il s'ensuive que Dieu a une chance sur deux d'exister. Il n'admet pas qu'une "entière ignorance à l'égard d'une réalité constitue par elle-même un renseignement précis sur cette réalité ${ }^{22}$. Le pari de Pascal ne reposait pas sur une présomption fondée, mais uniquement sur une possibilité logique. Il n'était pas rationnel. Pour cela, il aurait dû porter sur une possibilité réelle. Une chose est logiquement possible si on peut la concevoir sans contradiction. Mais elle est réellement possible si on connaît des « raisons positives » à son existence, c'est-à-dire « des causes

20. CEUVRES, II, p. 201.

21. Psychologie et métaphysique, pp. 208 et suiv.

22. Notes sur le pari de Pascal, GEUVRES, II, p. 52. 
déterminées à la produire ${ }^{23}$. N'entendons pas des causes efficientes, car il serait trop difficile d'assurer que toutes les conditions d'un événement sont ou seront réalisées. D'ailleurs y réussirait-on que celui-ci ne serait plus une possibilité, mais une nécessité. C'est du point de vue des causes finales, ou, ce qui est même chose, des genres et des espèces qu'une chose est jugée réellement possible. Seront telles les "spécifications virtuellement contenues dans un genre existant ${ }^{24}$. Par exemple, on peut dire réellement possible que l'enfant à naître soit un garçon, parce que la masculinité est virtuellement contenue dans l'humanité, qui est sa forme générale et comme sa $\mathrm{fin}^{25}$. Or y a-til une existence dont cette vie corporelle et la vie éternelle seraient à concevoir comme deux spécifications ? Qu'on regarde en soimême.

Chaque fois que nous pensons ou que nous voulons, nous trouvons « en notre conscience un élément singulier . . . qu'on peut appeler à peu près indifféremment raison ou liberté $"{ }^{26}$. Ce quelque chose ne se donne jamais séparé d'un phénomène psychologique, représentation ou inclination, qui est comme sa matière ; mais la "réflexion " peut " l'isoler et le saisir en luimême ${ }^{27}$. Nous constatons alors la disproportion, et même la contradiction qu'il y a entre la forme et la matière de ces actes intérieurs. Considérons un jugement : l'usage même de la copule être indique que l'intention, la forme de mon acte est de dire ce qui est vrai pour toute intelligence ; mais en fait, qu'ai-je exprimé d'autre qu'un point de vue ? Considérons une volonté : je veux en principe ce qui pour la pure raison est le meilleur ; je veux en fait ce que mes représentations et mes inclinations me donnent pour tel. "La raison et la volonté débordent donc manifestement notre conscience actuelle : elles sont en nous l'idée en partie indéterminée, le cadre à moitié vide d'une vie spirituelle qui ne se réalise que très imparfaitement en ce monde et qui pourrait se réaliser beaucoup mieux dans un autre, si, tous les éléments sensibles de la conscience ayant disparu, la matière des

23. Ibid., p. 49 .

24. Ibid., p. 50.

25. Ibid., p. 51.

26. Ibid., p. 53 .

27. Ibid., p. 53. 
actes intellectuels devenait adéquate à leur forme ${ }^{28}$. Ainsi, c'est la tension entre l'idéal de la raison pure et son ravalement dans notre individualité qui met à même de saisir en nous, exigence très haute et toujours offensée, la réalité du genre esprit. Voilà pourquoi la vie ultraterrestre est une possibilité réelle. "Elle est la spécification éventuelle d'un gente dont nous saisissons en nous-mêmes l'existence ${ }^{29}$.

Dans ces conditions, l'enjeu du pari, qui est le sacrifice des biens liés à notre individualité, n'excède pas le risque encouru, puisqu'il y a vraiment quelque chance pour que l'esprit se détache du corps et quelque chance pour qu'il s'évanouisse avec lui. L'acte de foi va certes au delà de la raison, mais, loin de s'y opposer, il satisfait son effort en dessinant l'horizon depuis lequel toute expérience humaine est pensable. "La question la plus haute de la philosophie, dit Lachelier, plus religieuse déjà peutêtre que philosophique, est le passage de l'absolu formel à l'absolu réel et vivant, de l'idée de Dieu à Dieu. Si le syllogisme y échoue, que la foi en coure le risque, que l'argument ontologique cède la place au pari ${ }^{30}$. Croire, c'est donc parier qu'après cette vie mixte il y a "la vie de la pensée pure " ${ }^{31}$. Le risque n'est pas aveugle, puisqu'une telle vie serait l'accomplissement et d'une virtualité constatable et de la suprême aspiration : la foi est " rationnelle et raisonnable ${ }^{32}$.

Récapitulons : la critique kantienne enlève au spiritualisme traditionnel la méthode d'introspection et le support démonstratif de l'affirmation de l'au-delà ; mais elle introduit, sur le rapport du sujet et de l'objet, une conception inédite qui, une fois supprimés les noumènes, devient le centre d'une doctrine renouvelée de l'esprit ; par ailleurs, l'affirmation de l'au-delà, bien que renvoyée à la croyance religieuse, est l'épilogue souhaitable à l'acte formellement philosophique. Ainsi a pensé, si je ne me trompe, l'initiateur de l'idéalisme classique en France.

À mon avis, la rencontre du spiritualisme avec l'idéalisme ne fut pas aussi heureuse. Sans doute, philosophie de l'esprit,

28. Ibid., p. 54.

29. Ibid., p. 54.

30. Ibid., p. 56.

31. S.F.P., 19 novembre 1908, EUVRES, II, p. 164.

32. Ibid., p. 165. 
l'idéalisme offrait un cadre conceptuel à l'idée de la primauté de la conscience, comme à celle du fond rationnel des choses. Mais pour l'autre article du spiritualisme, pour l'au-delà, un important problème de cohérence me semble devoir être noté.

Pour le mettre en lumière, je considérerai successivement ce que l'on peut regarder comme les deux volets de l'affirmation de l'au-delà chez Lachelier : d'abord l'affirmation de la vie éternelle, ensuite celle du Dieu transcendant. Par delà l'exemple de ce philosophe, je crois que sera concernée la métaphysique postkantienne en général.

Examinons encore par quelle voie Lachelier a pensé mettre au jour dans la conscience humaine un sujet d'immortalité où le pari religieux pût prendre appui. Comment prend-il connaissance de ce que, dans son étude sur le pari de Pascal, il appelle l'esprit ? Je rappelle ses expressions : "Il y a dans notre conscience un élément singulier "; " cet élément nous est donné ", non « à l'état pur », mais " combiné avec un phénomène sensible "; " par un effort de réflexion ", nous pouvons "l'isoler et le saisir en lui-même "; l'esprit est " un genre dont nous saisissons en nous-même l'existence ${ }^{33}$. Cette réflexion par laquelle nous pouvons isoler l'élément spirituel et le saisir en lui-même est certainement une activité abstractive, qu'on doit distinguer de la simple introspection. Mais pour concevoir ainsi l'esprit à l'état pur, il a bien fallu l'éprouver à l'état mixte, il fallu l'expérimenter. Sous peine que la réflexion porte à vide, c'est à ce niveau de conscience préalable, celui de l'expérience, même impure, que nous « saisissons en nous-mêmes " l'existence de l'esprit. Donc l'expérience interne, ou l'introspection, est bien, dans l'analyse de Lachelier, ce qui fonde la connaissance en nous de l'esprit qui survit. Mais il faut se souvenir des objections qu'il avait lui-même dirigées contre la méthode d'introspection : ce qui se présente à ma conscience psychologique comme spirituel, ou libre, l'est-il vraiment ? Qu'est-ce qui m'assure qu'un simple fait psychique soit quelque chose d'original par rapport au corps ? S'il n'en tenait qu'à l'expérience, « comment pourrions-nous savoir si un sentiment ou une volonté existe réellement . . . et distinguer un véritable fait de conscience d'une

33. Notes sur le pari de Pascal, p. 53-54. 
illusion du sens interne ${ }^{34}$ ? " Il faut déduire l'action spirituelle $»^{35}$. Et c'est bien ainsi que la doctrine avait établi l'esprit : comme principe immanent de l'existence et de la connaissance des phénomènes, et dont l'essence spirituelle réside dans sa totale spontanéité. Seulement, cet esprit, tout entier à l'être de la nature, n'était pas susceptible de s'en détacher. "La nature, disait Schelling, est l'esprit invisible ; l'esprit est la nature invisible. "

C'est dire que les développements qui prétendent donner appui au pari religieux en accréditant l'évidence introspective d'un élément incorporel contredisent non seulement la méthode, mais l'idée centrale de la doctrine. Pour celle-ci, l'esprit c'est l'être, l'être vrai des phénomènes, entendus comme termes médiateurs dans l'acte de s'apparaître, qui est à lui-même son sujet. Rappelons-nous ces mots : " Je ne crois pas qu'il faille parler de sujet absolu ni de pensée universelle comme si la pensée préexistait aux choses ou pouvait être quelque chose en dehors d'elles. La pensée, c'est la vérité, et la vérité est dans les choses elles-mêmes. C'est là que l'esprit doit se chercher s'il veut se trouver . . . ${ }^{36}$. Or comment accorder cette thèse avec celle d'un esprit en soi, qui existerait selon la formule inconnue d'une substance au cœur de l'étendue, quoiqu'indépendamment et en négation d'elle ? Cet esprit serait une âme cartésienne. Mais cette entité sans rapport aux phénomènes n'est pas concevable, on l'a vu. "La réalité immatérielle de l'homme n'est pas anima ou animus : elle est mens, dira Brunschvicg ${ }^{37}$. L'esprit défini par l'idéalisme ne peut pas plus se démarquer du monde que la pensée ne peut se retrouver au delà d'elle-même. Voilà pourquoi, indépendamment des raisons particulières à Lachelier, il ne semble pas qu'une élaboration philosophique dans le contexte d'un idéalisme absolu puisse fournir le moindre contenu à l'idée d'un esprit qui survit au corps. On doit plutôt dire que si l'esprit survit au corps, sa conception idéaliste ne vaut pas.

Contrairement à la thèse mi-religieuse, mi-philosophique de la survie, l'affirmation de Dieu transcendant n'a recours à

34. Psychologie et métaphysique, p. 184.

35. À Paul Janet, 12 décembre 1887 , Lettres, p. 140 , cité plus haut.

36. S.F.P., 25 février 1904, GUVRES, II. P. 126, cité plus haut.

37. Spinoza et ses contemporains, P.U.F., 1951, p. 279. 
aucun préalable doctrinal. Elle se présente entièrement comme un discours de croyance. À peine Lachelier fait-il voir le caractère «désintéressé » et, en ce sens, "raisonnable » de cette croyance $^{38}$. Et sans doute, dans la mesure où elle ne demande rien à la doctrine philosophique, elle ne risque pas de la compromettre. Mais justement, la croyance religieuse ne prend-elle rien à la doctrine?

La compatibilité de la pensée religieuse et du système de Lachelier a été l'objet, chez les commentateurs, d'un désaccord assez partagé $e^{39}$. Ce qui sera critiqué, à travers la tentative de ce philosophe, c'est la supposée pente théologienne de l'idéalisme, c'est l'idée que la métaphysique postkantienne se prolonge naturellement en discours sur Dieu.

$\mathrm{Si}$, dans le système de Lachelier, contrairement à celui de Kant, on attribue pleine réalité aux contenus et principes de la connaissance, c'est, comme j'ai essayé de le montrer, qu'il n'existe rien en dehors de la pensée universelle. Sont réelles les qualités sensibles qu'on a raison de sentir. Plus réel est l'intelligible qui se manifeste en elles et les légitime, par exemple la nécessité causale qui s'extériorise en enchaînements de phénomènes. Plus réelle, c'est-à-dire plus a priori que la causalité efficiente est la finalité qui s'y détaille ${ }^{40}$. C'est en remontant ainsi de principe en principe qu'on déduit enfin, mais sans le voir, cet être tout extase qui est la source et le retour des choses, la pensée qui se pense en pensant le monde. Mais avec la position d'un réel qui transcende la pensée, il n'est plus vrai, évidemment, que la pensée soit identique à l'être. C'est même à cet au-delà, à cet impensable, que désormais convient le mot être. Les principes

38. S.F.P., 19 novembre 1908 , CEUVRES, II, pp. 163 et suiv.

39. Pensent qu'il y a incompatibilité : Gabriel Séailles (La pbilosopbie de Jules Lachelier, pp. 158 et suiv.), Régis Jolivet (De Rosmini à Lachelier, Vitte, 1953, p. 81), Louis Millet (Le symbolisme dans la philosophie de Lachelier, pp. 240 et suiv.). Pensent qu'il y a compatibilité et même complémentarité : Charles Dévivaise ("La philosophie religieuse de Lachelier ", Revue des sciences philosophiques et théologiques, 1939, pp. 435-464), Henry Duméry (Philosophie de la religion, P.U.F., 1957, I, pp. 284 et suiv.), Jacques Chevalier, (Histoire de la pensée, Flammarion, 1966, IV, pp. 446 et suiv.), Édouard LeRoy (communication à la S.F.P., Bulletin, 28 mai 1932, pp. 143-150).

40. Voir $D u$ fondement de l'induction, ch. 6, CEUVRES, I, pp. 68-81. " Les vraies raisons des choses, ce sont les fins, qui constituent, sous le nom de formes, les choses elles-mêmes : la matière et les causes ne sont qu'une hypothèse nécessaire, ou plutôt un symbole indispensable, par lequel nous projetons dans le temps et dans l'espace ce qui est, en soi, supérieur à l'un et à l'autre ». (p. 80). 
a priori de la connaissance subissent auprès de Dieu le sort auquel les condamnaient les noumènes de Kant. Ils sont ramenés au même non-lieu ontologique, pour ainsi dire à la même inexistence. Et il en ira de même pour l'univers entier, pour tout ce qui dans le système était concevable comme émanation de la pensée. Chez Kant, que les premiers principes fussent pures fonctions logiques, cela laissait intact un dehors pourvoyeur de matière phénoménale. Derrière l'objet, il y avait l'être, qui pour lui-même ne devait rien au sujet. Mais si cette origine de l'expérience n'est plus, si le sujet, en plus de déterminer le phénomène, le constitue dans sa matérialité, s'il est donc le principe de ce en quoi consistera l'être, rien n'existera si le sujet n'existe pas. Dieu, ou quoi que ce soit au delà de la pensée, est bien la réalité en regard de laquelle le monde seulement représenté n'est pas réel. Il est l'être qui disqualifie l'ontologie idéaliste. S'il y a l'en-soi, de quelle réalité est ce qui est en nous, qui sommes dans l'Idée, qui n'est pas dans l'en-soi ? La pensée religieuse de Lachelier, avons-nous dit, ne compromet pas le système dans la mesure où elle n'y prend rien. Elle y prend quelque chose cependant : la valeur d'être.

Examinons maintenant si ce non-être du transcendantal devant l'en-soi peut être dépassé du fait que Lachelier définit comme objet de sa croyance un Dieu chrétien, créateur du ciel et de la terre. Dieu " pose le monde, dit-il, non comme nous le posons nous-mêmes, par une action purement idéale, qui n'est que la reconnaissance d'une réalité déjà donnée, mais dans son fond, par une action efficace et productrice de réalité, par un " fiat " . . . qui est un fait " ${ }^{41}$. La pensée ainsi ramenée à l'être, à titre de créature, peut-être semblera-t-il que le discours religieux redonne ici au système ce qu'en se constituant il lui a prélevé. On admettra toutefois, à supposer que la position d'un Dieu créateur ait cette portée, que la pensée universelle, alors, n'est plus aussi spontanée. Elle devient une substance pensante, en puissance devant son existence et devant son acte. Il n'y a plus le pur acte de penser, mais sous lui une potentialité qui ne s'extasie pas, un substrat irréductible qui ne sera ni matière ni forme de phénomènes. À nouveau l'apparaître n'est pas l'être ;

41. S.F.P., 19 novembre 1908, CEUVRES, II, p. 160-61. 
d'autant que les phénomènes, ou plutôt les corps, eux aussi créés par Dieu, dans l'extension d'un même concept d'être, et qui s'ils n'étaient que déterminations du cogito substantiel n'existeraient pas vraiment, les corps seront forcément extérieurs à la connaissance, maintenant "reconnaissance d'une réalité donnée ». Si Dieu crée le monde "dans son fond ", "par une affirmation efficace et productrice de réalité ", sans doute il ne se peut plus que l'affirmation nôtre, au sein de la pensée universelle, constitue "l'acte créateur lui-même, absolument efficace en même temps que purement idéal $" 42$. Il ne faudra pas répéter que la nature est " complète en elle-même " et que "l'au-delà et à son égard comme s'il n'était pas " ${ }^{43}$. La pensée devant l'en-soi n'était plus qu'idée. Devant le Dieu créateur, cette idée même se détruit.

L'idéalisme, était-il ressorti, ne laisse aucun sens à la proposition : l'esprit survit au corps. Il faut ajouter que la simple hypothèse de l'au-delà religieux est deux fois celle de sa contradiction. S'il y a l'Être sans rapport à notre rationalité productrice de phénomènes, ce monde des phénomènes n'est pas véritable ; si, plutôt, l'Être se communique au monde, alors notre rationalité devient participation ou créature, et elle ne produira pas plus les phénomènes qu'elle ne se produira ellemême. Dans un cas se trouve compromise toute métaphysique, dans l'autre, toute métaphysique idéaliste. L'ontologisme de la pensée vraie ne survit à rien qui lui échappe, ni au transcendant, ni, plus généralement, à ce qui se distinguerait de la matière et de la forme de la connaissance objective.

Que l'idéalisme soit spiritualiste, je le tiens donc pour à moitié vrai. Certes, s'il s'agit de dire que l'esprit est réel, la doctrine qui nie la nouménalité est déjà un immatérialisme, et elle est spécifiquement un spiritualisme dans la thèse que le pensable est un acte absolu de manifestation, où l'esprit connaissant est le pôle naturant, c'est-à-dire l'alpha et l'oméga. Seulement, ce schéma d'une épiphanie circulaire de la forme ne se

42. À Frédéric Rauh, 19 mars 1893, Lettres, p. 155, cité plus haut.

43. S.F.P., 19 novembre 1908, EUVRES, II, p. 164. 
maintiendra que sur le fond d'un néant infini. Que survienne donc l'ens realissimum! L'idéalisme n'est ni théiste ni déiste, parce que l'être-idée qu'il conçoit, l'être de Dieu l'annihilerait, comme le soleil les ombres de la Caverne.

Département de philosophie Université d'Ottawa 